\title{
Optical System based on Multiplexed FBGs to Monitor Hand Movements
}

\author{
Abian B. Socorro-Leranoz, Silvia Diaz, Silvia Castillo, Uilian J. Dreyer, Cicero Martelli, \\ Jean Carlos C. da Silva, Itziar Uzqueda, Marisol Gomez, Carlos R. Zamarreño
}

\begin{abstract}
This contribution reports the development and characterization of an optical system based on parallel Fiber Bragg Gratings (FBGs) to monitor the movements of the wrist and fingers of a hand. The system consisted of a reflective configuration made of FBGs detecting the movements of the fingers and one more located on the wrist as a reference. All FBGs were multiplexed in order to collect the basic movements of the hand. Fibers were embedded in polydimethylsiloxane for protection and to give flexibility to the optical detection setup. Measurements of strain, angle and torsion were performed during the experiments, obtaining sensitivities up to $1.29 \mathrm{pm} / \mu \varepsilon$ in strain and $64.23 \mathrm{pm} /{ }^{\circ}$ in angle. Also, a study on the influence of a single sensor on the performance of the whole system was analyzed for a complete study of this proof of concept. The obtained results present a simple system that can be used to monitor the positions of the hand or for the rehabilitation of patients suffering from neuromotor or post-stroke diseases.
\end{abstract}

Index Terms- Biomechanics; Fiber Bragg gratings; Fiber-optic sensors; Polydimethylsiloxane (PDMS); Hand posture

\section{INTRODUCTION}

$\mathrm{O}$ $\mathrm{NE}$ of the current major challenges in biomedical engineering is the development of biomechanics. This discipline tries to mimic the mechanical parts of our organism, such as the lower and upper limbs, the musculoskeletal system or the cardiac valves, just to mention some of the most interesting working areas. The imitation of the precise movements involved in each specific biomechanical application implies to deal with a complex state of the art, where electronics, anatomophysiology, biocompatibility and the own mechanical operation of the organism must be synchronized so that everything works properly.

A very interesting example of such complexity is the analysis of hand posture and movements [1], the focus of this work. Human beings use our hands for almost everything. Trying to imitate these movements is crucial nowadays, since it is possible to create prostheses that can replace hand basic functions in case someone loses them for any reason or even

Manuscript received April 02, 2020; revised June 08, 2020.

This work was supported by both Spanish and Brazilian research projects. From the Spanish side, the funding came in part by the National Research Agency through the project with reference TEC2016-78047-R and also by the Government of Navarra project with reference SENSMOV: 0011-1365-2017-000135. From the Brazilian side, the financial support came in part from Fundação Araucária, Secretaria de Estado da Ciência, Tecnologia e Ensino Superior do Paraná (SETI), CNPq, FINEP, and also by the Coordenação de Aperfeiçoamento de Pessoal de Nível SuperiorBrazil (CAPES)-Finance Code 001.

A.B. Socorro-Leranoz, S. Diaz, S. Castillo and C.R. Zamarreño are with the Dept. of Electrical, Electronic \& Communication Engineering and also with the Institute of Smart Cities, both at Universidad Publica de Navarra (UPNA), 31006 - Pamplona, Navarra, Spain (corresponding author e-mail: ab.socorro@unavarra.es).

U.J. Dreyer, C. Martelli and J.C.C. da Silva are with the Federal University of Technology - Paraná, Curitiba- 80230-901, Brazil.

I. Uzqueda and M. Gomez are with the Dept. of Statistics, Informatics \& Mathematics and also with the Institute for Advanced Materials, both at UPNA, 31006 - Pamplona, Navarra, Spain. needs help for rehabilitation. That is why hand prosthetics (or bionics) is one of the most developed areas in biomechanics.

From a commercial point of view, the website 'Wareable' reported in 2017 on the existence of eight startups developing bionics to help people around the world gain life quality [2]. Companies like Open Bionics (openbionics.com/), Mobius Bionics (mobiusbionics.com/) or Ossur (ossur.com), among others, are developing cutting-edge bionic hands capable of not only moving the different joints involved in a hand, but also even imitating the sense of touch, for both children and adult customers.

In the meanwhile, there are also different approaches from the research point of view. One of the main focuses here is the modelling of hand biomechanics [3], by means of the development of an optimized sensing network around the hand. In this way, it is possible to know the position of the fingers and the generated forces in every point instantly. Such kind of sensors can be made of different technologies, but the most used are those based on accelerometers and gyroscopes. These simple sensors can be attached anywhere on either the hand or a glove covering the hand in order to know the position of the fingers [4] or the turns of the wrist [5], [6]. The corresponding data are taken and then processed for both prosthetics or rehabilitation purposes [7].

The present work addresses this issue by using light and, specifically, the light propagated inside an optical fiber. As it is well-known, it is common to find sensing applications where optical fibers are used. Temperature [8], strain [9] or bending [10], among others, are good examples of typical parameters that can be detected with this technology. However, using the optical fiber cable for biomedical purposes makes sense too, since it offers interesting properties to be considered when it is introduced in the organism. Some of them are biocompatibility, reduced size, flexibility, weight and cost or capability to work within complex matrices media, such as the blood [11], [12]. Moreover, it is also possible to 
detect chemical and biochemical reactions, just by depositing proper nanomaterials onto these waveguides [13]. This has led to the detection of different kinds of biomolecules, even at very low limits of detection [14], [15]. Apart from that, optical fibers are nowadays quite common in medicine due to their use in endoscopy, to burn varicose veins [16] or to perform surgery for prostate hyperplasia [17], among other applications.

Regarding biomechanics, the main focus of this article, the fact of being sensitive to strain or bending makes the optical fiber suitable to address this issue, since it is possible to transduce mechanical variables into light modulation. Some examples are reviewed in [18], where several optical configurations based on fiber Bragg gratings (FBG) are used to measure strain in bones, map the pressure in orthopaedical joints, measure the stress between the intervertebral discs or the chest wall deformation, or even estimate the forces induced by tendons and ligaments. In these works, the wavelength shift of the FBG's Bragg peak is tracked as a function of the different efforts.

Talking about hand movements, posture and gestures, some contributions can be found reporting the use of FBGs for these purposes. One of the most representative is written by Ferreira da Silva et al. [19]. Authors present an optical fiberbased glove made of 14 FBGs sequentially distributed along the fingers, with 3 FBGs per finger measuring the movements of every phalanx joint. FBGs are embedded within an almost 1-micron thick three-layer PVC glove. The designed software is capable of obtaining the shift in wavelength as a function of the flexion/extension of an FBG. Generally speaking, the same measurements are obtained by Jang et al. in their contribution some years after [20]. In this case, they distinguish between shape and angle FBG-based sensors, so that they can accurately calculate the relative position between the index and thumb fingers when pinching by means of software. There is a third strategy, which is the use of "hetero-cored" or "multi-cored" optical fibers [21]. Here, Nishiyama et al. propose fusing two fibers with different core diameters, so that an interferometry is generated between the involved light propagation modes. As the flexion angle of the finger varies, so does the wavelength location of the visualized spectral bands. Therefore, it is simple to translate an angular movement into an optical wavelength shift. Finally, one of the most recent contributions is the one written by Jha et al. [22]. Here, the authors, based on the previous references, develop a robotic system that moves the FBGs automatically, at the same time they can measure the FBG bending with interesting values of sensitivity, accuracy and precision.

Despite the interest of the previous contributions reporting FBGs for hand biomechanics, most of them show what happens in only one or maybe two sensors. Just a few characterize the behavior of the FBGs once inside the prototype and none of them analyze the possible influence of a single FBG on the others, especially when these FBGs are located one after the other. In the end, the fact of straining several FBGs in series will affect to those sensors directly subjected to this action, but also to the closest FBGs. This may mask the actual performance of the whole system. Unless these measurements are adequately characterized, there will be a lack of accuracy during the experiments.
In this sense, this contribution will try to fill these gaps generated by the technology so far. First, a simple optical configuration will be proposed, to monitor the fingers and wrist movements. Second, a characterization of the prototype behavior as a function of strain (elongation and flexion angle) will be shown. Then, an analysis of the influences that may exist between the FBGs located on fingers and wrist while moving them will be presented. Finally, the corresponding conclusions will be summarized in order to encourage the scientific community to keep improving the capabilities of this FBG-based technology when used in biomechanics.

\section{EXPERIMENTAL CONDITIONS}

\section{A. Optical setup}

The optical setup used in this contribution to track the hand movements is shown in Fig. 1. The FBG monitoring was performed by the I-MON system from Ibsen Photonics A/S. The light source model DL-BP1-1501A is a super-luminescent diode source (SLED) suitable for FBG interrogation, with a bandwidth of $70 \mathrm{~nm}$ in the $1550 \mathrm{~nm}$ wavelength range, which also contains an optical circulator. It consists of a compact plug-n-play module and its power can be regulated through a user interface. As the interrogating element, the IBSEN USB I-MON 512 series model was used, with an accuracy of $5 \mathrm{pm}$ and a wavelength fit resolution close to $0.5 \mathrm{pm}$.

In addition to the interrogation hardware, I-MON includes a software with its own interface running on LabVIEW ${ }^{\circledR}$. This software is capable of monitoring the reflection spectra from the FBGs in real time, by communicating via the PC's USB port. The graphical interface of the interrogator allows to detect where the reflected FBG peaks are spectrally located and their corresponding wavelength shift. Additionally, the provider also includes the source libraries of its software to develop a customized application based on it. An adaptation of this software was done in order to obtain the results on the screen at the same time they were collected.

A total of six FBGs with resonant wavelengths centered at $1514.98,1523.06,1530.45,1544.04,1552.68$ and $1540.55 \mathrm{~nm}$

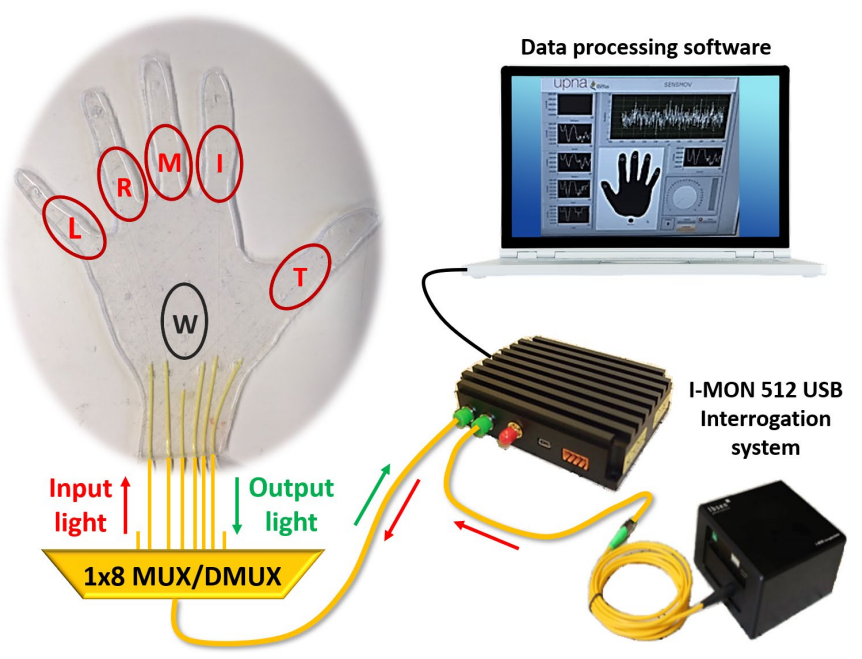

Fig. 1. Fiber-optic-based optical interrogator system designed for measuring the performance of the PDMS-embedded 6-FBG sensing platform. The location of the FBGs on the wrist (W), thumb (T), index $(\mathrm{I})$, middle $(M)$, ring $(R)$ and little $(L)$ fingers is indicated in the figure. 
were used to monitor the signals reflected from thumb (T), index (I), middle (M), ring (R) and little (L) fingers and the wrist (W) respectively. The wrist FBG was used as a reference for the rest. Consequently, only relative measurements were obtained during the experiments. The FBG signals were combined by a 1 x8 multiplexer/demultiplexer (MUX/DMUX). This allowed the introduction of the light along the FBGs at the same time that the reflected signals from every FBG could be collected at the input of the interrogator.

\section{B. Embedding of FBGs in PDMS and additional instrumentation}

Usually, these applications require the encapsulation of the FBGs in materials such as polyvinyl chloride (PVC) [19], polyimide (PI) [23] or polydimethylsiloxane (PDMS) [24], [25]. The objective is to guarantee some protection and avoid an excess of torsion, strain or bending that ends up breaking the sensors. In this work, PDMS was used, since it is considered one of the most suitable materials due to its interesting properties, such as the fact of being visually transparent, non-toxic, non-flammable and biologically inert. Moreover, PDMS stands out for its durability, great flexibility, low cost and simplicity when prepared. It can be demolded easily, and its rigidity can be regulated using different proportions of its main components [26].

PDMS is a silicone-based material, whose consistency varies depending on the size of its polymer chains. More specifically, a PDMS with short chains looks like liquid, whereas a long-chain PDMS is more rigid. Usually, PDMS is acquired in liquid state and it is necessary a curing agent to harden it. Once these two substances are mixed, the solidification takes place by means of crosslinking. In this work, a mixing ratio 10:1 was used. This means, 1 part of curing agent and ten of the polymer, a ratio suggested by Sylgard ${ }^{\mathbb{B}} 184$ from Dow Corning.

The PDMS mixture was dropped into a 3D-printed plastic mold with the shape of a hand for curing. The FBGs were then introduced, supported by several small 3D-printed grooves in the mold, which allowed the suspension of the fibers inside the matrix. Every FBG was located so that it coincided over each metacarpophalangeal joint on top of the hand. The reference FBG (W) was placed centered slightly over the wrist, as depicted in Fig.1. Once here, all the components were subjected to a thermal treatment at $55^{\circ} \mathrm{C}$ for 4 hours in a vacuum oven, in order to degas the polymer and decrease the solidification time [26]. These steps permitted the FBGs to be embedded inside the PDMS matrix with apparently no adhesion issues.

The fact of monitoring six FBGs in parallel should not be addressed without testing the resistance and the robustness of the proposed configuration. That is why some more embedded FBGs were manufactured and then subjected to two kinds of tests. The first one was to fix some embedded FBGs to a micropositioner (model M-UMR8.51 from Newport Corporation) and strain them. The linear stages of this micropositioner allowed a displacement ranging from 0.2 in. $(5 \mathrm{~mm})$ to 3.15 in. $(80 \mathrm{~mm})$, with $0.1 \mu \mathrm{m}$ steps. This instrumentation helped check the sensitivity of the PDMSembedded FBGs to strain. Also, a measuring system based on two goniometers fabricated by Movalsys S.L. and an angle protractor were used. The goal here was to estimate the behavior and the limitations of the embedded FBGs when subjected to different angles.

\section{Results AND Discussion}

\section{A. Sensitivity to elongation/strain}

The first approach to evaluate the operation of the proposed system was to test the sensitivity of the PDMS-embedded FBGs to elongation, as depicted in Fig. 2a. The experiment consisted of inducing a total displacement of $300 \mu \mathrm{m}$, taking data every $50 \mu \mathrm{m}$ elongation step applied, and retaining that position for $10 \mathrm{~s}$. This process was repeated 10 times. Then, all the measurements were imported to Origin ${ }^{\circledR}$ for processing. The procedure was based on calculating the medians of the data in every step and subtracting them to the median of the data in the reference position. The reason for using the median instead of the mean is that the median is more significant, since it is normally used as a bias between the half of the data that can be considered as unstable (they present higher variation) and the lower half of the data where the signal tends to stabilize [27].

Data processing leads to Fig. 2b. Here, the evolution of the FBG peak wavelength as a function of time for every 50micron step through one of the 10 cycles during the test is shown. A maximum shift in wavelength of $350 \mathrm{pm}$ is registered between the initial and final positions, showing an almost instant response between steps. Then, after processing the 10 cycles along the experiment, a linear regression was performed to obtain Fig. 2c. The Pearson coefficient after the adjustment was $\mathrm{R}^{2}=0.99996$, indicating a linear relationship between the two variables. This means that there is a high correlation between the wavelength shift and the elongation of the material with negligible data dispersion. Consequently, the sensor presents high repeatability. As indicated in the chart, the attained sensitivity is $1.29 \mathrm{pm} / \mu \varepsilon$.

\section{B. Sensitivity to angle variations}

The metacarpophalangeal joint subjected the PDMSembedded FBG to angular variations, due to its flexion extension movements. Therefore, this second experiment was intended to obtain the response of the embedded FBGs when subjected to these angle variations. As occurred in section III.A, the hypothesis was that a variation of the metacarpophalangeal joint angle was translated into a variation in the fiber elongation, leading to a wavelength shift of the FBG peak.

To carry out this experiment, a real situation was tested. The 6-FBG sensing platform was placed on top of a latex glove where the hand of an individual was previously introduced (see Fig. 3a). As commented in section II, two goniometers were attached to this proof of concept on the metacarpus and index finger phalanx respectively. This experiment was also repeated for 10 times. Every test started from the initial resting situation, where the individual was seated, with the elbow flexed at approximately $90^{\circ}$, and supporting the forearm, wrist and fingers in their anatomical position. Then, the index finger was flexed by increasing intervals of $5^{\circ}$, starting and returning to the original resting 

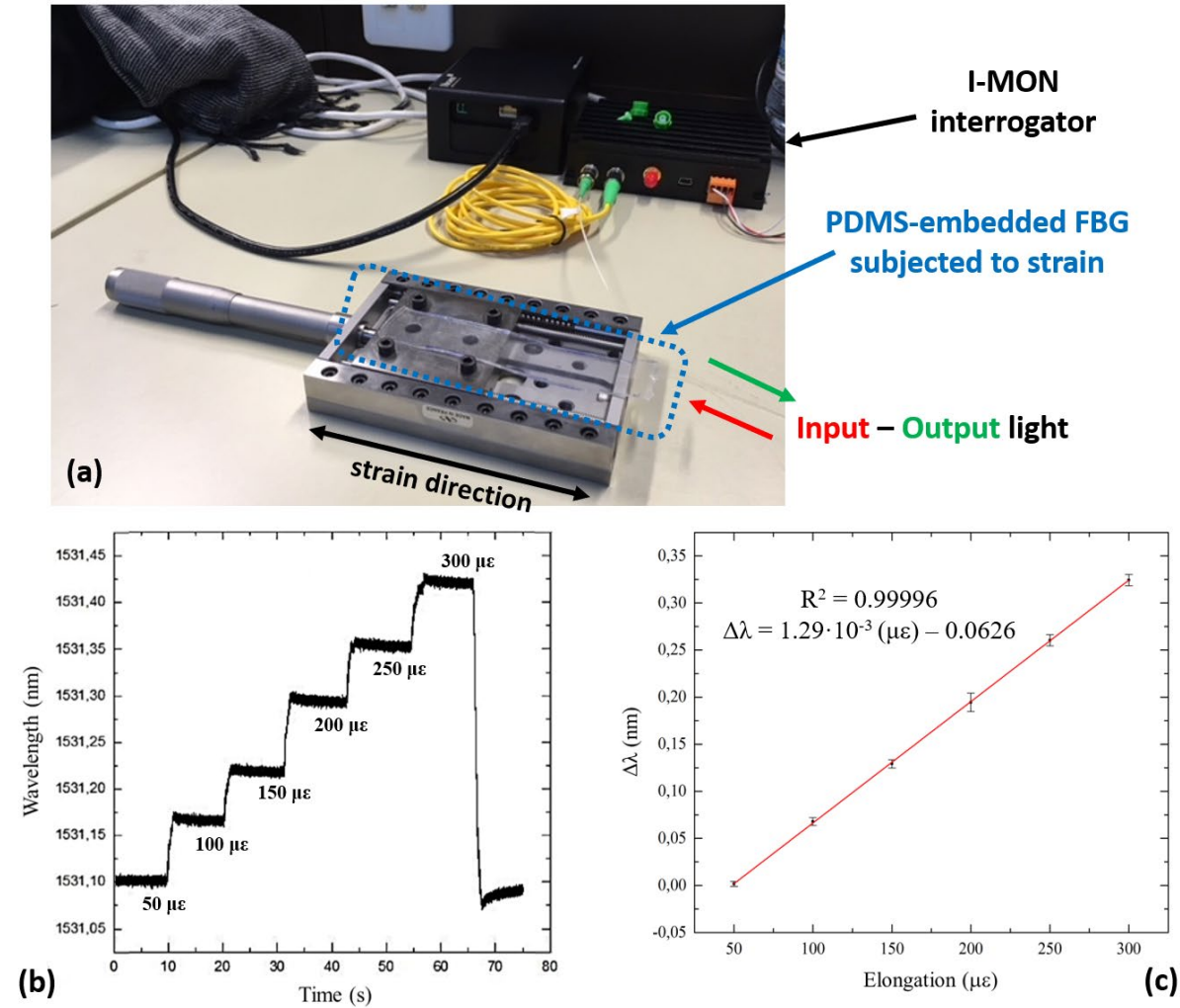

Fig. 2. (a) Optical setup prepared for the characterization of a single PDMS-embedded FBG when subjected to strain by means of a micropositioner. (b) Time response of the sensor as a function of the increasing elongation. (c) Linear regression of the obtained results to obtain the elongation sensitivity.

situation at $0^{\circ}$. Each testing interval took between $5-10 \mathrm{~s}$. The angle measurement interval ranged from $0^{\circ}$ to $35^{\circ}$. Again, the collected data were imported to Origin ${ }^{\circledR}$. Both goniometric and wavelength shift values were then processed by median calculations for the same reasons in section III.A.

Fig. $3 \mathrm{~b}$ shows the behavior of the PDMS-embedded index sensor (I) as a function of the flexion angle. The response of the goniometer has been also introduced with a short delay to facilitate the comparison between signals. The shape of both goniometric and optical curves is quite similar. However, a slight non-linear behavior is obtained with the optical setup, which is more noticeable for the higher angle measurements. Consequently, a higher red-shift in wavelength is registered on the FBG resonant peak, leading to an increase in the estimated angle. Also, it can be observed that every angle increment makes the optical response present a transient before reaching its actual final value. The explanation to this behavior comes from the own embedded FBG. Flexing the finger involves straining the FBG. Moreover, the FBG is inside a PDMS matrix, which gives elasticity to the sensing part of the system. Once the finger has reached the desired bending angle, there is an adaptation process of the PDMS-embedded FBG due to the mentioned elasticity that can be monitored, since the sensitivity of the FBG can detect slight adjustments on the bending. At the same time, the FBG is also capable of detecting the vibrations of the finger as a fruit of maintaining the finger position. This can be seen as a little ripple in the signal before stabilizing. It is also noticeable that, as the bending angle increases, the strain induced on the embedded
FBG is higher than desired and this is probably translated into a measurement error. However, in the case of the goniometers, they are not subjected to strain, so they cannot measure the adaptation to the new induced angle. Consequently, their response is more stable.

The previous optical results are summarized in Fig. 3c. It is noticeable that a maximum wavelength excursion of $2.5 \mathrm{~nm}$ is registered when subjecting the fibers to angle variation, with a sensitivity of $64.23 \mathrm{pm} /{ }^{\circ}$. Moreover, and despite the quite instantaneous and exact results obtained in the previous analysis, in this case the data dispersion increases as the metacarpophalangeal joint angle increases. A slight difference can be noticed between their estimations, with a Pearson coefficient of $\mathrm{R}^{2}=0.99148$.

\section{Assessment of system performance}

After checking the sensitivity of the PDMS-embedded FBG to elongation and flexion/extension angle, the main goal was to assess the general behavior of the optical system, test each sensor individually and check the influence of moving a single finger or the wrist with respect to the rest of sensors, this means, the cross-talk between FBGs. No absolute angles or displacements were considered here. That is why relative measurements will be provided henceforward. All the measurements were taken using the optical system described in section II and based on Fig. 3a.

Regarding the monitoring of the wrist FBG sensor (W), three types of movements were monitored: flexion-extension, radial - ulnar deviation and pronation - supination, as 

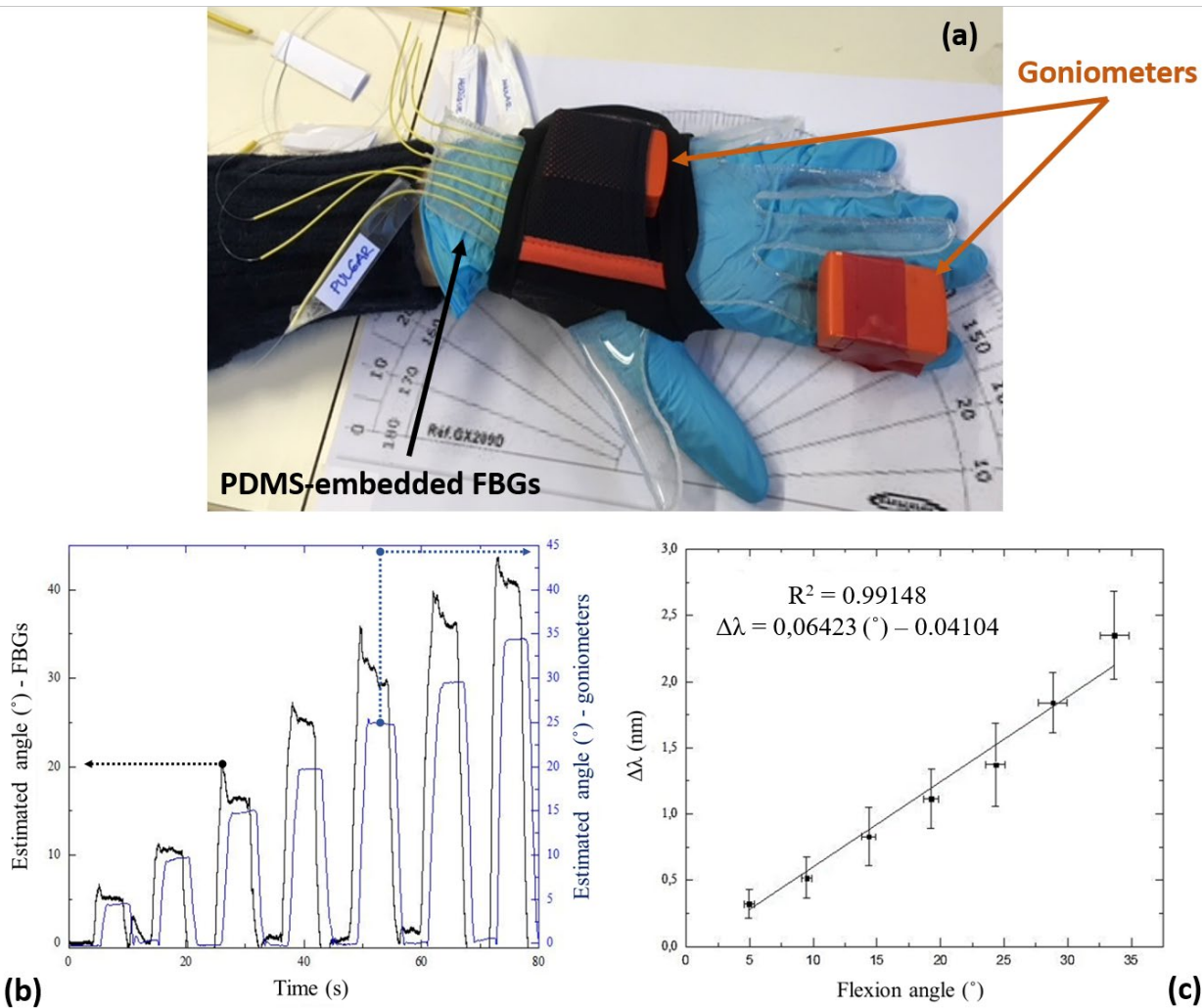

Fig. 3. (a) Optical setup prepared for the characterization of the angular response of the index FBG (I). (b) Time estimation of the reconstructed angles by means of the FBG-based optical system designed (black line) and goniometers (blue line). (c) Regression of the obtained results.

indicated in Fig. 4a. In all the cases, the movements started from the rest position, in which the person is seated, with the elbow flexed at approximately $90^{\circ}$, and supporting the forearm wrist and fingers in their anatomical position. Again, once the data were collected, they were transferred to Origin ${ }^{\circledR}$. In each movement interval, the tracking of the wavelength shift for every FBG peak was collected by calculating its median and the difference between the maximum variation acquired and the reference value of the initial wavelength (resting situation).

Fig. $4 \mathrm{~b}$ depicts the behavior pattern of the wrist movement, repeating every set of movements five times. Several aspects are worth commenting. First, the flexion-extension movements produce higher wavelength shift than in the rest of cases. This is consistent with the fact that the elongation takes place along a direction favorable to the strain of the FBG, so all the force is transferred directly to the sensor as elongation up to $1 \mathrm{~nm}$ shift. After that, the radial - ulnar movement is performed. As it can be observed, the effect of the lateral movement is not so accused than in the case of the flexion extension. In spite of that, it is possible to detect an overall wavelength shift of $0.6 \mathrm{~nm}$. Regarding the pronation supination, turning the wrist implies a more noticeable wavelength shift, since the torsion applied to the fiber may vary the position of the W-FBG more than in the case of radial - ulnar deviation. Again, this is probably due to the fact of rotating the fiber around its propagation axis.

After analyzing the W-FBG behavior, focus will now be centered on the influence of its movements on the rest of FBGs. As it can be observed in Fig. 4c, moving the wrist involves a considerable alteration of the wavelength shift for all the sensors located in the fingers. Both flexion - extension and radial - ulnar deviation movements induce wavelength shifts in a range between 1 and $2 \mathrm{~nm}$ maximum. Since all FBGs are embedded within the same PDMS matrix, the fact of moving the wrist strains all the fibers. This is something intuitive from an anatomical point of view, since flexing the wrist involves contracting the metacarpophalangeal tendons [1]. Also, it is remarkable that $\mathrm{I}$ and $\mathrm{M}$ responses are more noticeable than $\mathrm{R}$ and $\mathrm{L}$, with up to $2 \mathrm{~nm}$ and hardly $1 \mathrm{~nm}$ respectively. Depending on the flexibility of the individual's wrist, the amplitude of the movements that can affect to $\mathrm{R}$ and $\mathrm{L}$ tendons and, therefore, to $\mathrm{R}$ and $\mathrm{L}$ FBGs, is limited in comparison to the capacity of M and I FBGs, so in principle it is logical to obtain a lower response. Lastly, pronationsupination movements, suppose wavelength shifts up to 1.5 $\mathrm{nm}$, but the signals are not so clear and sometimes chaotic. The main reason is that turning the wrist provokes strain but also torsion to the fibers. Consequently, the response is not so clear, since there are other modes that can interfere with the reflected light, leading to signal distortion. In view of these results, it can be said that the presented sensing platform can check which fingers are more active and which are less, with a good distinction of the wrist movements.

Finally, a cross-talk analysis between a single finger FBG and the rest of sensors is performed. In this sense, Fig. 5 helps visualize the same trends observed so far. For each FBG located on each metacarpophalangeal joint, several repetitions of flexion - extension movements were performed, returning 


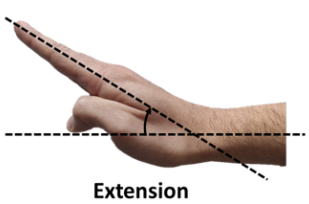

(a)
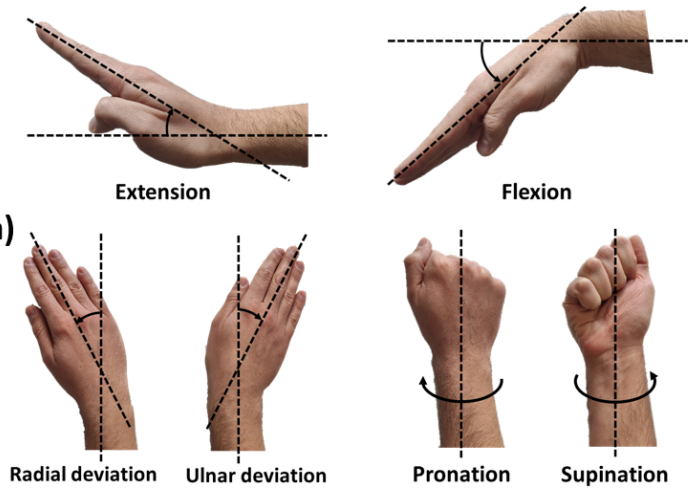

Flexion

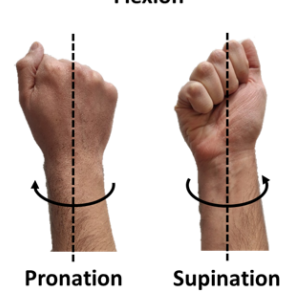

(b)

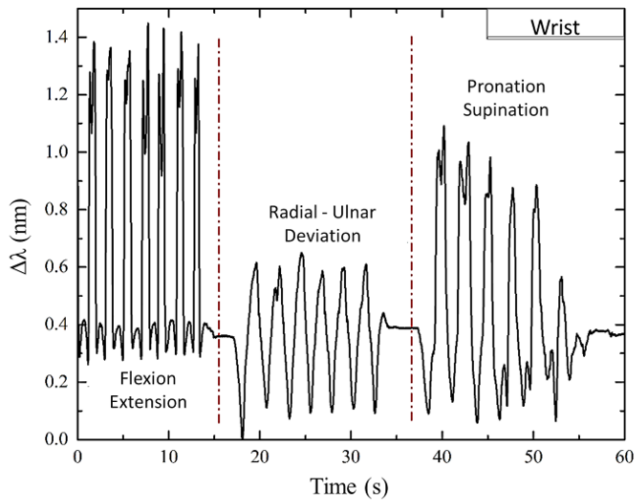

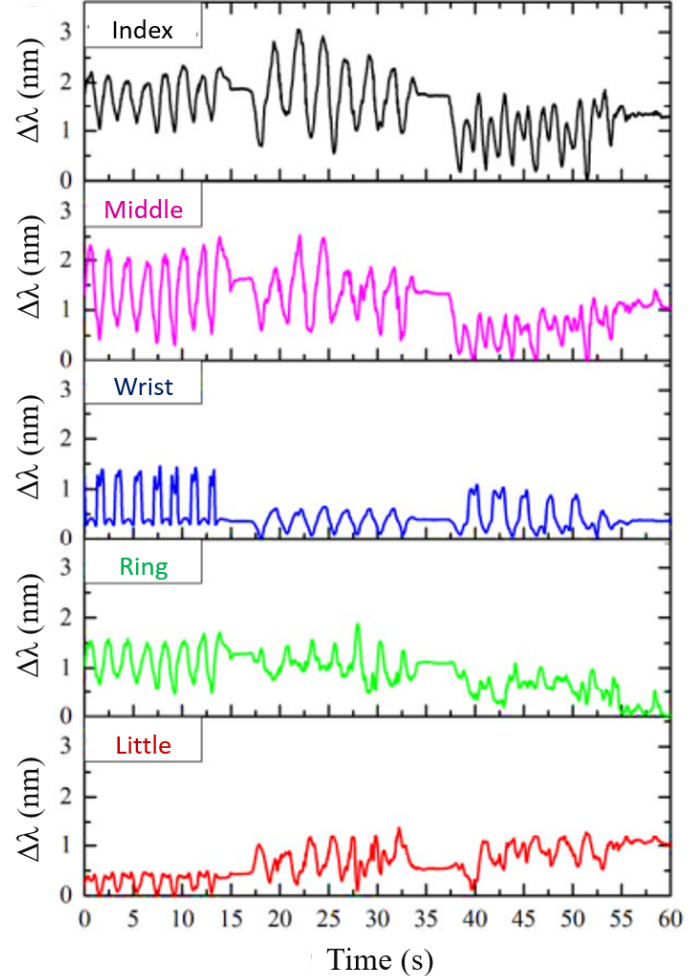

(c)

Fig. 4. (a) Sequence of movements performed during the wrist experiment. (b) Optical response of the W sensor as a function of time for the corresponding sets of movements. (c) Influence of the W sensor on the rest of FBGs.

to the rest point afterwards (see Fig. 5a and 5b). In the case of flexing the I FBG (Fig. 5c), this movement affects to the M FBG mainly, although middle finger oscillations are clearly lower $(0.35 \mathrm{~nm}$, half of the I FBG). The rest of fibers hardly notice what is happening on the index finger. The same can be affirmed regarding the M FBG: $1 \mathrm{~nm}$ wavelength shift on $\mathrm{M}$ FBG involves $0.5 \mathrm{~nm}$ in the I FBG (see Fig. 5d) and a very little effect on the rest of fingers.

In Fig. 5e, it can be observed that flexing the R FBG for up to $1.3 \mathrm{~nm}$ involves affecting both I and M FBGs $(0.3 \mathrm{~nm})$, with an almost null effect on the W and L FBGs. Finally, based on the results provided by Fig. $5 f$, it is noticeable the close relationship between the movements of the little finger and their effect on the middle finger. The L FBG presents flexions with oscillations of $1 \mathrm{~nm}$ that let the M FBG vary on the same order without moving the fingers. In spite of that, most sensors seem to be affected by the L FBG movements.

After this analysis, it seems clear that moving a specific FBG has a direct influence on those FBGs corresponding to the fingers that do not have a total independence on their mobility. The proposed platform can detect the recruitment of the necessary areas in the hand to produce a finger movement. This means, the real physiology of an individual's hand. This is positive, from the authors' point of view, since this means that it is possible to measure actual movements in the hand, not just a single movement due to a specific FBG.

At this point, it is interesting to visualize the operation of the presented FBG-based system. In this sense, V1 is attached as supplementary material to support the affirmations of this contribution. Inside the file, a sequence of movements is performed: basically, 4 extensions of I, M and W FBG sensors respectively. The designed software is capable of detecting which sensor is moving, the influence of this movement on the rest of FBGs and also the vibrations that retaining the hand in a desired position supposes for each one of the sensors involved. As it can be observed in the demonstration, the 5 fingers can be monitored in the graphs on the left and in the upper graph. While the upper graph represents the red/blue shift in wavelength as a function of the extension/flexion angle, the graphs on the left correspond to the time responses of the FBGs (in this case, the T sensor has been disconnected). Moreover, W FBG sensor is monitored on the right side, down the spectral response. The hand figure showing up on the center is programmed with simple umbralization algorithms. Basically, if the sensors have been adequately chosen and characterized, it is easy to estimate their maximum variation. As long as the wavelength shift of each individual FBG does not overcome a determined range, it is possible to know what FBG is being subjected to an effort and what kind of effort. This effect is especially noticeable in the last part of the video, where the W FBG is tracked. It can be checked that the W FBG peak red-shifts while extending and blue-shifts while returning to the original position, this means, when flexing. This kind of movements are quite typical for training patients who have lost mobility. due to ictus episodes or either need for rehabilitation sessions to recover the neural control of their hand nerves. In opinion of the authors, this system could be an interesting alternative for these purposes. 
(a)

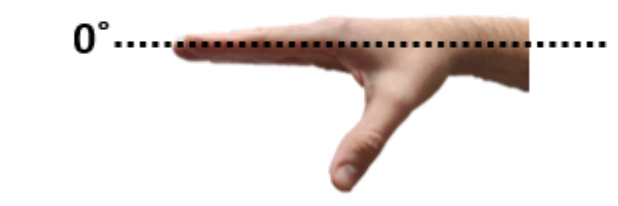

(c)
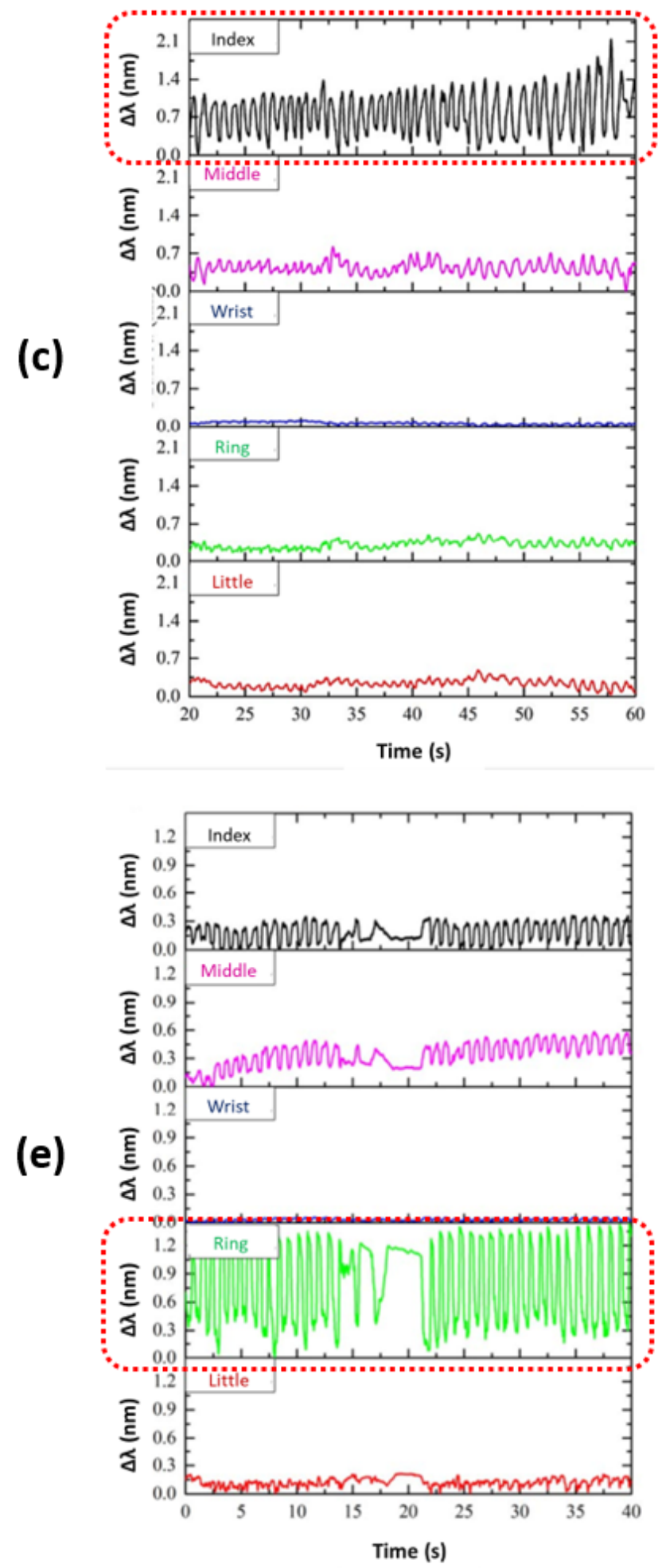

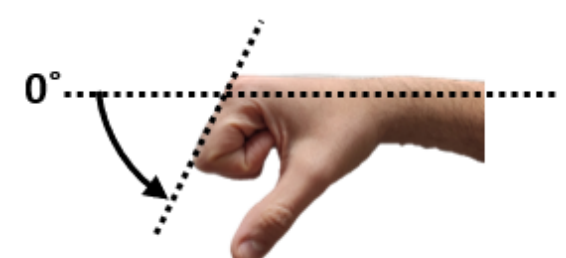

(b)

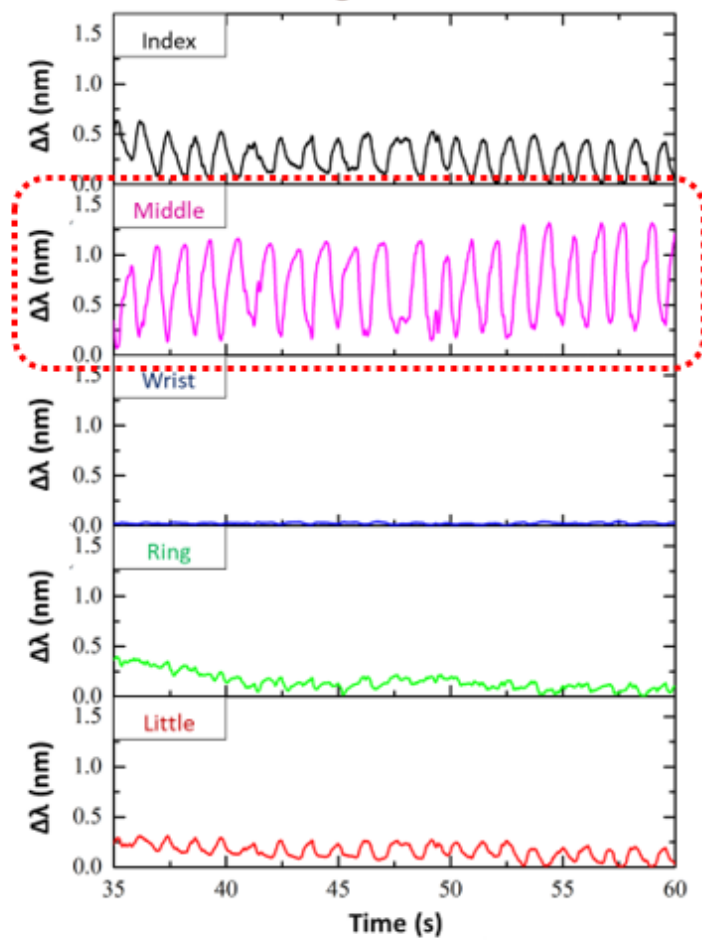

(d)

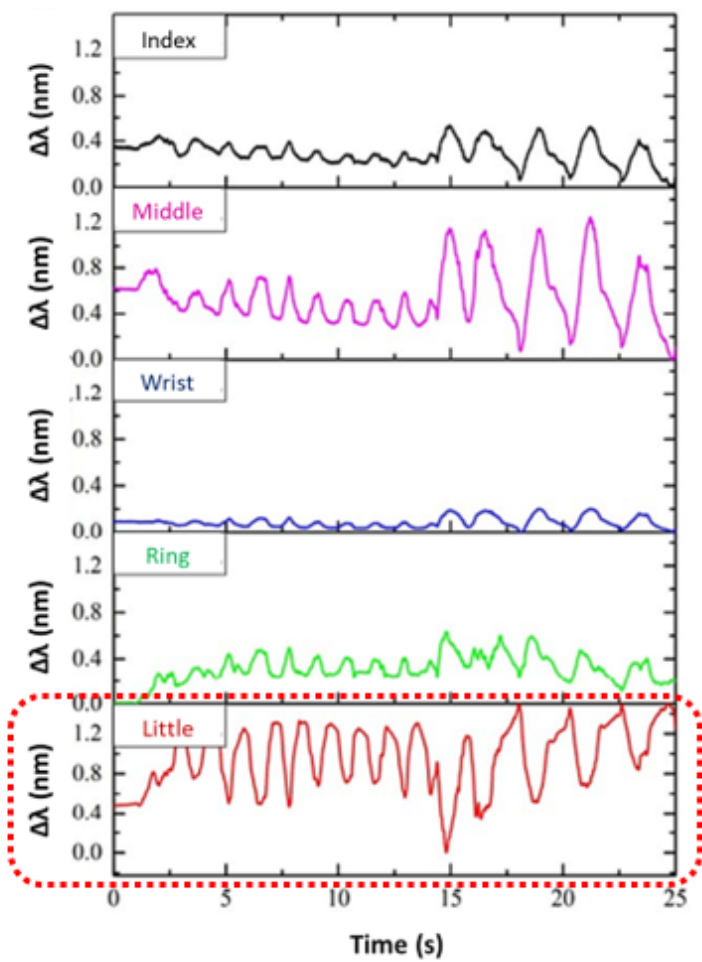

Fig. 5. (a) and (b) Sequence of movements performed during the monitoring of the metacarpophalangeal - wrist experiment. (c) to (f) Optical response of the finger sensors to check their influence on the rest. In most cases, the wrist sensor is not influenced by the fingers' movements. Just the opposite situation when moving the wrist and checking the fingers. 


\section{CONCLUSIONS}

Several conclusions can be extracted after showing all the experiments performed inside this work. First, a fiber-optic sensing platform based on multiplexed FBGs has been designed and characterized in order to detect the movements of both the wrist and the fingers of a hand. The fact of placing the FBGs aligned with the tendons makes it possible to detect and track their movements in real time. Also, it is possible to detect the direction and the strain range for basic movements such as flexion - extension, radial - ulnar deviation and pronation - supination.

The fact of embedding the FBGs in a polymer matrix such as PDMS facilitates the transduction of the movement to the FBG by means of the Young modulus of the polymer. This has been checked when subjecting the PDMS-embedded FBG to microdeformations or to angle variations. Sensitivities up to $1.29 \mathrm{pm} / \mu \varepsilon$ and $64.23 \mathrm{pm} /{ }^{\circ}$ have been obtained respectively. Apart from that, embedding several FBGs within the polymer helps check the influence of a single FBG on the response of the rest. Moving a specific finger involves the recruitment of several tendons and muscular fibers. This configuration can detect these real hand movements.

Finally, further work should be done in order to improve the features of this proof of concept. Due to the mechanical limitations in the optical setup, the implemented system detects deformations generated by angles between 0 and $35^{\circ}$. This could be enough for patients with reduced hand mobility. However, it is obvious that there is a need for more flexibility, in order to include a higher amplitude of movements, at least from 0 to $90^{\circ}$. Another improvement to address is temperature compensation. FBGs are typical examples of fiber-optic temperature sensors. No studies related to this issue are known in the revised literature. However, it could be interesting to check whether temperature has an actual effect on the behavior of these systems. Lastly, a deeper analysis should be done to understand how this system behaves with a higher number of patients. Different responses should be expected for different people, due to the diversity of movements associated to an individual's physiology. In this sense, this initial prototype may help develop future studies to check the mobility pattern of patients under rehabilitation process.

\section{REFERENCES} of the hand," Hand Clin., vol. 29, no. 4, pp. 483-492, 2013. L. Johnson, "Beyond human: 8 organisations making bionic breakthroughs," Wareable, 2017. [Online]. Available: https://www.wareable.com/wearable-tech/organisations-makingbionic-breakthroughs-1411. [Accessed: 20-Mar-2020].

[3] C. E. Hrabia, K. Wolf, and M. Wilhelm, "Whole hand modeling using 8 wearable sensors: Biomechanics for hand pose prediction," ACM Int. Conf. Proceeding Ser., pp. 21-28, 2013.

[4] N. Bhatt and S. K. M. Varadhan, "Posture similarity index: A method to compare hand postures in synergy space," PeerJ, vol. 2018, no. 12, 2018.

[5] P. Z. Chen, J. Li, M. Luo, and N. H. Zhu, "Real-time human motion capture driven by a wireless sensor network," Int. J. Comput. Games Technol., vol. 2015, 2015.

[6] H. Han and S. W. Yoon, "Gyroscope-based continuous human hand gesture recognition for multi-modalwearable input device for human machine interaction," Sensors (Switzerland), vol. 19, no. 11, 2019.

[7] A. F. Panaite, M. N. Rişteiu, M. L. Olar, M. Leba, and A. Ionica,
"Hand rehabilitation- A gaming experience," IOP Conf. Ser. Mater. Sci. Eng., vol. 572, no. 1, 2019.

[8] S. Silva et al., "Ultrahigh-sensitivity temperature fiber sensor based on multimode interference," Appl. Opt., 2012.

[9] A. Abdallah, "Experimental study on an interferometric strain sensor based on hollow-core photonic bandgap fiber for intrusion detection," Opt. Commun., vol. 428, no. July, pp. 35-40, 2018.

[10] Hariyanti, K. Devara, F. N. Aisyah, K. V. A. W. Nadia, and R. W. Purnamaningsih, "Design of a wearable fiber optic respiration sensor for application in NICU incubators," AIP Conf. Proc., vol. 2092, no. April, 2019.

[11] X. D. Wang and O. S. Wolfbeis, "Fiber-Optic Chemical Sensors and Biosensors (2013-2015)," Anal. Chem., vol. 88, no. 1, pp. 203-227, 2016.

[12] F. Chiavaioli, F. Baldini, S. Tombelli, C. Trono, and A. Giannetti, "Biosensing with optical fiber gratings," Nanophotonics, vol. 6, no. 4. pp. 663-679, 2017.

[13] A. B. Socorro-Leránoz, D. Santano, I. Del Villar, and I. R. Matias, "Trends in the design of wavelength-based optical fibre biosensors (2008-2018)," Biosens. Bioelectron. X, vol. 1, no. February, p. $100015,2019$.

[14] F. Chiavaioli et al., "Femtomolar Detection by Nanocoated Fiber Label-Free Biosensors," ACS Sensors, vol. 3, no. 5, pp. 936-943, 2018 .

[15] P. Zubiate et al., "Fiber-based early diagnosis of venous thromboembolic disease by label-free D-dimer detection," Biosens. Bioelectron. X, vol. 2, no. August, p. 100026, 2019.

[16] P. W. M. Van Ruijven, A. A. Poluektova, M. J. C. Van Gemert, H. A. M. Neumann, T. Nijsten, and C. W. M. Van Der Geld, "Opticalthermal mathematical model for endovenous laser ablation of varicose veins," Lasers Med. Sci., vol. 29, no. 2, pp. 431-439, 2014.

[17] B. Chughtai, L. Laor, C. Dunphy, and A. Te, "Contemporary review of the $532 \mathrm{~nm}$ laser for treatment of benign prostatic hyperplasia," Asian J. Urol., vol. 2, no. 2, pp. 102-106, Apr. 2015.

[18] E. Al-Fakih, N. A. Abu Osman, and F. R. Mahamd Adikan, "The use of fiber Bragg grating sensors in biomechanics and rehabilitation applications: the state-of-the-art and ongoing research topics.," Sensors (Basel)., vol. 12, no. 10, pp. 12890-926, Sep. 2012.

[19] A. Ferreira Da Silva, A. F. GonÇalves, P. M. Mendes, and J. H. Correia, "FBG sensing glove for monitoring hand posture," IEEE Sens. J., vol. 11, no. 10, pp. 2442-2448, 2011.

[20] M. Jang, J. S. Kim, K. Kang, J. Kim, and S. Yang, "Towards Finger Motion Capture System Using FBG Sensors," Proc. Annu. Int. Conf. IEEE Eng. Med. Biol. Soc. EMBS, vol. 2018-July, pp. 37343737, 2018.

[21] M. Nishiyama and K. Watanabe, "Wearable sensing glove with embedded hetero-core fiber-optic nerves for unconstrained hand motion capture," IEEE Trans. Instrum. Meas., vol. 58, no. 12, pp. 3995-4000, 2009.

[22] C. K. Jha, A. L. Chakraborty, and S. Agarwal, "A Fiber Bragg Grating-based Sensing Glove with a Sensitivity of $18.45 \mathrm{pm} /$ degree to Accurately Assess Finger Flexure," in 26th International Conference on Optical Fiber Sensors, 2018, p. WB4.

[23] T. L. Yeo, T. Sun, K. T. V Grattan, D. Parry, R. Lade, and B. D. Powell, "Characterisation of a polymer-coated fibre Bragg grating sensor for relative humidity sensing," Sensors Actuators B, vol. 110, pp. 148-155, 2005.

[24] G. T. Kanellos, G. Papaioannou, D. Tsiokos, C. Mitrogiannis, G. Nianios, and N. Pleros, "Two dimensional polymer-embedded quasi-distributed FBG pressure sensor for biomedical applications," Opt. Express, vol. 18, no. 1, p. 179, 2010.

[25] A. Santiago-Alvarado and S. Vázquez-Montiel, "Propiedades físicoquímicas de membranas PDMS empleadas en lentes líquidas," Superf. y Vacio, vol. 22, no. 3, pp. 61-66, 2009.

[26] C. N. Lafratta et al., "Replication of Two-Photon-Polymerized Structures with Extremely High Aspect Ratios and Large Overhangs," 2004.

[27] A. Bakker and K. P. E. Gravemeijer, "An historical phenomenology of mean and median," Educational Studies in Mathematics, vol. 62, no. 2. pp. 149-168, Jun-2006. 